\title{
Activation of spinal ERK1/2 contributes to mechanical allodynia in a rat model of postoperative pain
}

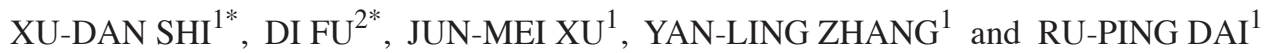 \\ ${ }^{1}$ Department of Anesthesia, The Second Xiangya Hospital of Central South University, Changsha, Hunan 410011; \\ ${ }^{2}$ Department of Anesthesia, Xiangya Hospital, Central South University, Changsha, Hunan 410078, P.R. China
}

Received November 4, 2012; Accepted February 22, 2013

DOI: $10.3892 / \mathrm{mmr} .2013 .1347$

\begin{abstract}
Extracellular signal-regulated kinase (ERK) 1/2 in the spinal cord has been implicated in the development of neuropathic pain and inflammatory pain. However, a limited number of studies have investigated the role of spinal ERK in incisional pain. The present study aimed to determine the role of ERK in the spinal cord in incisional pain. Incisional pain was established in rats by a unilateral hind paw incision. ERK1/2 expression was analyzed by immunohistochemistry. Hypersensitivity to pain was evaluated by measuring the paw withdrawal threshold using the von Frey test. The mitogen-activated protein kinase kinase (MEK) inhibitor, U0126, was administered $20 \mathrm{~min}$ prior to or $10 \mathrm{~min}$ following the incision by intrathecal or intraperitoneal injection. Phosphorylated ERK1/2 in the ipsilateral L4-5 spinal superficial dorsal horn was activated $1 \mathrm{~min}$ following the incision, reached its peak level at $5 \mathrm{~min}$ and then returned to the basal level $20 \mathrm{~min}$ following the incision. Pretreatment, but not post-treatment with U0126 markedly attenuated the pain hypersensitivity induced by the incision. Therefore, the present study indicates that the transient activation of spinal ERK1/2 contributes to the initiation of pain hypersensitivity following surgical incision.
\end{abstract}

\section{Introduction}

Extracellular signal-regulated kinase (ERK) is a member of the mitogen-activated protein kinase family and has been implicated in a number of biological functions, including learning and memory in the hippocampus and pain-related negative affects in the anterior cingulated cortex (1-3). Several studies have identified that ERK in the spinal cord contributes to the

Correspondence to: Dr Yan-Ling Zhang, Department of Anesthesia, The Second Xiangya Hospital of Central South University, 86 Ren-Min Road, Changsha, Hunan 410011, P.R. China E-mail: zhangyanling0618@yahoo.cn

*Contributed equally

Key words: pain, incision, extracellular signal-regulated kinase, spinal cord, allodynia induction and development of pain. Firstly, it was reported that spinal ERK is persistently activated in inflammatory pain induced by the injection of complete Freund's adjuvant into the hind paw and the inhibition of activated spinal ERK attenuated the development of mechanical hypersensitivity (4). Subsequently, it was reported that spinal ERK is sequentially activated in neurons, astrocytes and microglia in rat models of neuropathic pain (5). Pretreatment or post-treatment with an ERK inhibitor through intrathecal delivery markedly inhibited or reversed the mechanical allodynia and thermal hyperalgesia following neuropathy (5). These observations indicate that the persistent activation of spinal ERK regulates chronic pain. However, few studies have investigated whether spinal ERK is involved in surgical pain processing.

Compared with chronic pain, surgical pain is more transient and requires specific perioperative pain management $(6,7)$. Compared with inflammatory and neuropathic pain, surgical pain is a unique acute pain state in which there are various central sensitization mechanisms, in particular in the spinal cord. The $N$-methyl-D-aspartate (NMDA)-dependent mechanism regulates the development of neuropathic and inflammatory pain. However, it has been reported that the NMDA-independent but not the NMDA-dependent mechanism mediates the mechanical pain hypersensitivity induced by incision, a surgical pain mode (8). In addition, a previous study revealed that administration of tumor necrosis factor (TNF) IgG fusion protein did not attenuate the mechanical hypersensitivity induced by a surgical incision (9). These observations indicate that TNF does not contribute to incisional pain behavior, although TNF is known to regulate other forms of persistent pain (10). The distinct neurochemical changes in the spinal cord following the surgical incision indicate that the role of ERK in surgical pain may be different from that in other types of pain. Therefore, the present study aimed to explore the role of ERK in the spinal cord in mechanical hypersensitivity following surgical incision.

\section{Materials and methods}

Animals. Adult male Sprague-Dawley rats (150-250 g) obtained from Central South University Animal Services (Changsha, China) were used in this study. All rats were kept in an air-conditioned $\left(23-26^{\circ} \mathrm{C}, 60-70 \%\right.$ relative humidity) vivarium with a $12 \mathrm{~h}$ dark/light cycle (light on from 8:00 am 
to $8: 00 \mathrm{pm})$. The experimental protocol complied with the National Institutes of Health Guide for the Care and Use of Laboratory Animals and was approved by the Animal Care and Use Committee of Central South University. All efforts were taken to minimize the suffering of the rats.

Surgical preparation and groups. The incisional pain model was established by unilateral hind paw incision. A detailed description of this surgical model in rats has been described previously (11). Our preliminary study observed that inhaled anesthetics (isoflurane or sevoflurane) induced the immediate expression of spinal ERK. Therefore, $10 \%$ chloral hydrate $(30 \mathrm{mg} / \mathrm{kg}$ ) was used as an anesthetic when examining ERK expression in the rats shortly after the establishment of incisional pain (1, 2, 5 and $10 \mathrm{~min})$. At later times $(1,3$ and $6 \mathrm{~h}$ and 1 and 3 days), $1.5 \%$ sevoflurane was used to anesthetize the animals as described previously (1). This was due to the short-term anesthetic effect of sevoflurane, which leads to the rats regaining consciousness within minutes. In brief, a $1-\mathrm{cm}$ long longitudinal incision was made into the planta skin and through to the plantaris muscle. The muscle was then elevated and incised longitudinally $(0.5 \mathrm{~cm})$. The shin was then closed by 4-0 nylon sutures. A topical triple antibiotic ointment was applied to the hind paw following surgery. Sham surgery was performed using the same procedure but with no incision.

For immunohistochemistry experiments, experimental rats were randomly divided and sacrificed at 1, 2, 5 and $10 \mathrm{~min}$, 1 and $6 \mathrm{~h}$ and 1 and 3 days ( $n=8-10$ for each group). In an independent group, rats were implanted with an intrathecal catheter for intrathecal delivery or intraperitoneal injection to enable the effects of inhibitors on ERK expression and pain behaviors to be investigated.

Intrathecal catheterization. In brief, a polyethylene-10 cathether was implanted in the intrathecal space of the spinal cord at the lumbar enlargement in a rat anesthetized with chloral hydrate $(30 \mathrm{mg} / 100 \mathrm{~g})(12)$. The mitogen-activated protein kinase kinase (MEK) inhibitor, U0126, was purchased from Sigma-Aldrich ( $1 \mu \mathrm{g}$ dissolved in 10\% DMSO; St. Louis, MO, USA). U0126 was intrathecally administered at 20 min prior to or 20 min following the incision and DMSO was injected as a vehicle control.

Immunohistochemistry. The rats were deeply anesthetized with chloral hydrate $(80 \mathrm{mg} / \mathrm{kg})$ and perfused transcardially with $100 \mathrm{ml}$ phosphate-buffered saline, followed by $4 \%$ paraformaldehyde in $0.1 \mathrm{M}$ phosphate buffer. L4-5 spinal cord segments were fixed for $4 \mathrm{~h}$ with $4 \%$ paraformaldehyde and then immersed in $20 \%$ sucrose in phosphate buffer ( $\mathrm{pH} 7.4)$ overnight. Transverse spinal cord sections $(30 \mu \mathrm{m})$ were cut and processed for immunohistochemistry using the ABC method. In brief, sections were mounted on 3-aminopropyl triethoxy-silane-coated slides and incubated with mouse anti-p-ERK antibody (dilution 1:1,000; Cell Signaling Technology, Danvers, MA, USA) at room temperature overnight. The secondary reagents used for localization were biotinylated goat anti-mouse IgG and an ABC kit (Vector Laboratories, Burlingame, CA, USA). Diaminobenzidine tetrahydrochloride (Sigma-Aldrich) was used as a peroxidase substrate.
Nociceptive testing. Mechanical allodynia was assayed by measuring the paw withdrawal threshold (PWT) using nylon von Frey filaments (13). In brief, the rats were placed on wire mesh platforms in clear cylindrical plastic enclosures and von Frey filaments (0.4-15.1 g) were applied to the edge of the wound in the incised hind paw or to the center of the plantar surface of the unincised paw. The up-down method was performed. The test was consecutive; in the absence of paw withdrawal response, a stronger stimulus was applied, otherwise a weaker stimulus was selected. Testing proceeded in this manner until four fibers had been applied after the first to cause a withdrawal response, allowing the estimation of the mechanical withdrawal threshold. Behavioral tests were performed prior to and at 10 and $30 \mathrm{~min}$ and 1,3 and $6 \mathrm{~h}$ until 5 days following incision.

Statistical analysis. Eight non-adjacent sections from each specimen of L4-5 lumbar spinal cord were randomly selected and p-ERK expression was determined by counting the p-ERK-positive cells on the L4-5 spinal superficial dorsal horn (lamina I and II). Data collection was performed by an individual who was blind to the treatments the animals had received. SPSS v13.0 (SPSS Inc., Chicago, IL, USA) and Prism 5.0 (Graphpad Software, San Diego, CA, USA) were used to perform statistical analysis. Data are presented as the mean \pm SEM. Differences between groups were compared with one-way or two-way ANOVA followed by post hoc Dunnett or Tukey post hoc multiple comparison tests where appropriate. $\mathrm{P}<0.05$ was considered to indicate a statistically significant difference.

\section{Results}

ERK activation in the spinal cord dorsal horn following hind paw incision. In sham-operated rats, low levels of p-ERK expression were identified in the dorsal horn of the spinal cord (Fig. 1A). However, 1 min following hind-paw incision, increased p-ERK immunoreactivity (IR) was detected within numerous dorsal horn neurons in the ipsilateral side (Fig. 1B and I). The induced p-ERK expression reached peak levels at 5 min post-incision (Fig. $1 \mathrm{C}$ and I), returned to baseline levels $10 \mathrm{~min}$ post-incision and remained at these levels thereafter (Fig. D-G and I).

A more highly magnified version of Fig. 1D revealed that the elevated p-ERK IR was localized in neurons in the cytoplasm of the soma and nucleus as well as in the neurites in the superficial dorsal horn (lamina I and II; Fig. 1H).

Effect of brushing the incised skin on expression of ERK at later times following incision. The transient activation of spinal ERK may be associated only with the initiation of incisional pain. However, activated spinal ERK is also involved in the maintenance of inflammatory and neuropathic pain (14). Our recent study demonstrated that ERK, in the anterior cingulated cortex, is reactivated by innocuous stimuli at the time when p-ERK expression has returned to basal levels during incisional pain (1). Since the mechanical allodynia induced by innocuous stimuli is clinically similar to the incident pain induced by coughing or moving and is a hallmark of postoperative pain, incised skin brushing was performed, as described previously, 

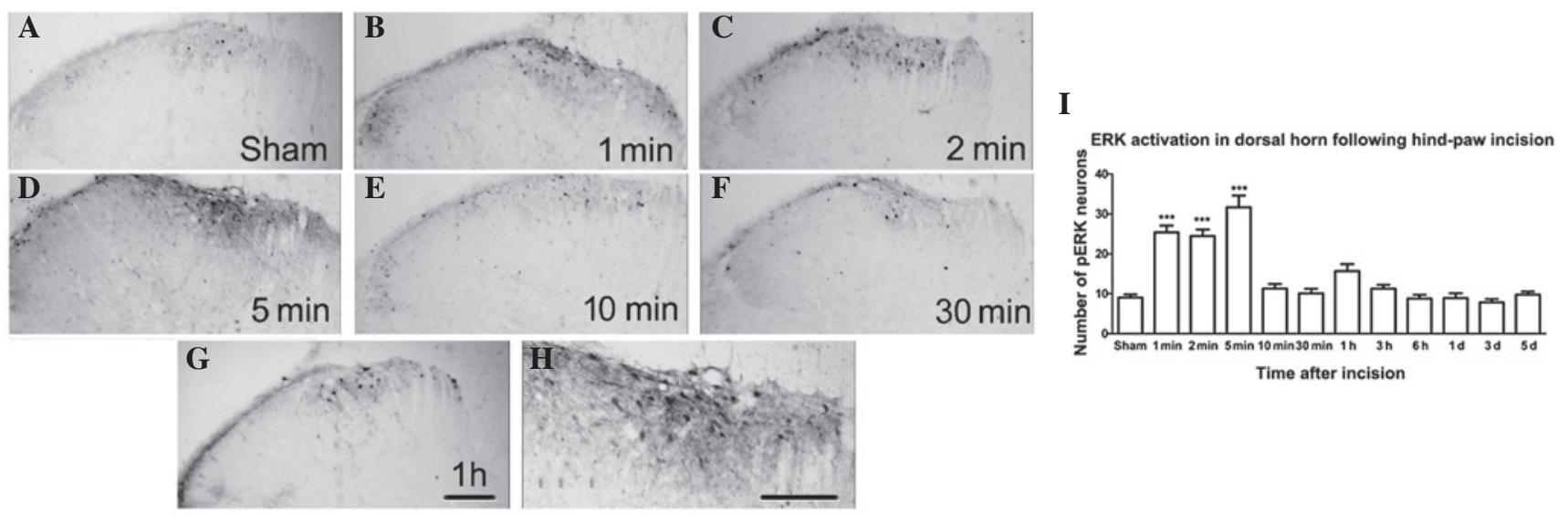

Figure 1. Activation of p-ERK in the dorsal horn following hind-paw incision. (A-G) Representative photomicrographs of L4-5 spinal cord segments revealing p-ERK IR in the ipsilateral superficial dorsal horn at various times following hind-paw incision and sham surgery. Surgical incision increased expression of p-ERK in the dorsal horn. Scale bar, $200 \mu \mathrm{m}$. (H) Higher magnification image of (D) revealing pERK-IR in a number of neurons in the superficial dorsal horn. Scale bar, $50 \mu \mathrm{m}$. (I) Time course of the number of $\mathrm{p}$-ERK-positive neurons in the ipsilateral side of the dorsal horn following hind-paw incision. ${ }^{* * *} \mathrm{P}<0.001$, vs. sham-surgery. One-way ANOVA followed by Dunnett post hoc test. p-ERK, phosphorylated extracellular signal-regulated kinase; IR, immunoreactivity.
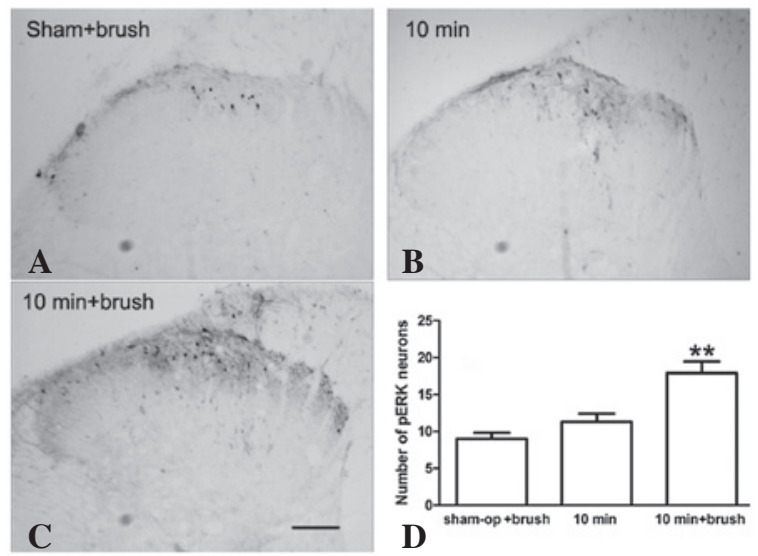

Figure 2. Effect of brushing on p-ERK expression in the dorsal horn. (A and B) Innocuous stimuli brushing had no effect on p-ERK compared with the 10 min group. (C) p-ERK expression in the ipsilateral superficial dorsal horn (lamina I and II) increased subject to brushing at $10 \mathrm{~min}$. Scale bar, $200 \mu \mathrm{m}$. (D) Brushing-evoked ERK activation, measured by the number of p-ERK-positive neurons. ${ }^{* *} \mathrm{P}<0.01$, vs. sham-surgery + brush and $10 \mathrm{~min}$ groups. Two-way ANOVA followed by Tukey post hoc test. Sham-op, sham-surgery; ERK, extracellular signal-regulated kinase; p, phosphorylated.

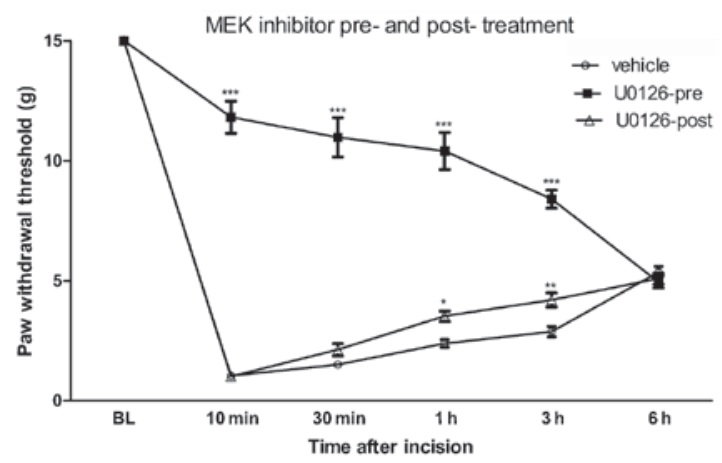

Figure 3. Pre- and posttreatment with MEK inhibitor, U0126, attenuated the pain behavioral response to hind-paw incision. U0126 $(1 \mu \mathrm{g})$ or vehicle (10\% DMSO) was administered 20 min prior to and following surgical incision, followed by the PWT test. ${ }^{* * *} \mathrm{P}<0.001$, U0126-pre vs, vehicle and U0126-post groups; ${ }^{*} \mathrm{P}<0.05$ and ${ }^{* *} \mathrm{P}<0.01$, U0126-post vs. vehicle groups. Two-way ANOVA followed by Tukey post hoc test. MEK, mitogen-activated protein kinase kinase; PWT, paw withdrawal threshold. and we examined whether innocuous stimuli reactivated p-ERK expression in the painful condition. As demonstrated in Fig. 2, p-ERK expression levels were markedly increased in the superficial dorsal horn (lamina I and II) with brushing compared with those in the incision+saline and sham-surgery groups. These observations indicate that the expression of p-ERK is activity-dependent at later times post-incision.

Effect of MEK inhibition on post-incisional pain hypersensitivity. The transient activation of ERK in the spinal cord following hind paw incision indicates that spinal ERK may be involved in the development of post-surgical pain. To test this hypothesis, a specific MEK inhibitor, U0126, was intrathecally delivered 20 min prior to or following the hind paw incision and the PWT was measured. Previous studies have revealed that $1 \mu \mathrm{g}$ U0126 is sufficient to block ERK activity and therefore $1 \mu \mathrm{g}$ U0126 was administered intrathecally in the present study $(1,4)$. As revealed in Fig. 3, pretreatment with U0126 attenuated the reduction in PWT from 10 min and up to $6 \mathrm{~h}$ post-incision.

By contrast, U0126 post-treatment had only a limited effect on the reduced PWT at the indicated times following hind paw incision (Fig. 3). In addition, a significant difference between U0126 pre- and post-treatment in the pain behavioral response to hind-paw incision was observed $(\mathrm{P}<0.001)$. These results indicate that the transient activation of ERK in the spinal dorsal horn contributes to the induction but not the maintenance of incision-evoked pain hypersensitivity.

\section{Discussion}

It is well known that spinal ERK is persistently activated and contributes to pain processing in chronic inflammatory or neuropathic pain (14). The present study reveals that spinal ERK is also activated in response to surgical incision, indicating that ERK may also be involved in the development of surgical pain. However, unlike inflammatory and neuropathic pain, the activation of spinal ERK in incisional pain is transient and returns to baseline levels rapidly (10 min post-incision). 
This indicates that there are distinct mechanisms of central sensitization for incisional pain and other types of pain. However, the immediate increase in p-ERK expression levels in neurons is similar to the early activation of p-ERK in neuropathic and inflammatory pain. In the neuropathic pain model, p-ERK expression in the spinal cord dorsal horns is sequentially activated in the neurons, microglia and astrocytes (5). The activation of neuronal p-ERK is also extremely transient and is sustained for $<6 \mathrm{~h}(5)$. The rapid activation of neuronal ERK in various types of pain may be due to direct burst firing induced by noxious stimuli. Consistent with this hypothesis, electrophysiological studies have identified that sustained $\mathrm{C}$-fiber stimulation is able to induce the activation of p-ERK in the spinal cord (15).

Of note, in the later phases of neuropathic pain, increased p-ERK expression largely occurs in activated glial cells (microglia and then astrocytes). Although our previous study also demonstrated that surgical incision induces the activation of microglia and astrocytes in the spinal cord, increased p-ERK expression was observed in microglia or astrocytes (16). One hypothesis is that surgical incision may not result in the robust activation of cytokines, in particular TNF- $\alpha$. With respect to this hypothesis, exogenous TNF- $\alpha$ may markedly induce p-ERK expression in neurons or glial cells. In addition, peripheral inflammation and spinal nerve injury lead to the robust activation of cytokines, including TNF- $\alpha$ and interleukin- $1 \beta$ (IL-1 $\beta$ ), in the glial cells (17-19). Inhibition of TNF- $\alpha$ may inhibit the activation of $\mathrm{p}-\mathrm{ERK}$ in the spinal cord in these types of pain model. However, TNF- $\alpha$ in the spinal cord is not activated in glial cells following hind-paw incision. In addition, blocking TNF- $\alpha$ does not attenuate the mechanical hypersensitivity in post-incisional pain. Together with our previous studies, the results of the present study indicate that surgical incision may induce milder neuroimmune responses in the spinal cord.

The transient activation of ERK in the spinal cord indicates that spinal ERK mainly contributes to the initiation, but not the maintenance of surgical pain. Indeed, the intrathecal administration of a MEK inhibitor, U0126, prior to spinal ERK activation (pretreatment) markedly attenuated the pain hypersensitivity following the incision. By contrast, when U0126 was administered after the return of p-ERK expression to baseline, a marginal analgesic effect on incision-evoked pain hypersensitivity was observed. However, U0126 pretreatment exerts an analgesic effect $>6 \mathrm{~h}$ after incision, indicating that blocking the induction of pain may also affect the subsequent maintenance of pain. This may be through the inhibition of noxious stimulus-evoked spinal synaptic long-term potentiation during central sensitization. Consistent with this hypothesis, a recent study revealed that the brief application of high doses of opioids not only reduces pain but also erases the spinal memory trace of pain (20).

In the present study, innocuous stimulation by brushing was found to reactivate the spinal ERK expression when it had returned to the baseline following incision. Pain evoked by innocuous mechanical stimuli in the incisional pain model mimics clinically incident pain (pain evoked by body movement or coughing), a hallmark of postoperative pain. Although pain during rest is generally easy to treat, incident pain remains a major challenge for postoperative pain control.
The reactivation of $\mathrm{p}-\mathrm{ERK}$ in response to brushing the incised skin indicates that p-ERK expression in the later phases of incisional pain is activity-dependent and associated with incident pain. Of note, our previous study demonstrated that surgical incision induced the upregulation of brain-derived neurotrophic factor (BDNF) in the spinal cord (21). Previous studies have reported that U0126 inhibits the upregulation of BDNF in inflammatory pain induced by Freund's complete adjuvant and neuropathic pain $(22,23)$. These observations indicate that BDNF-ERK signaling may contribute to incident pain in postoperative pain.

In conclusion, the present study reveals that surgical incision immediately induces the transient activation of ERK in the spinal cord. Transient activation of spinal ERK appears to mainly regulate the initiation, but not the maintenance of incisional pain as pretreatment but not post-treatment with the MEK inhibitor markedly attenuated incision-evoked pain hypersensitivity. The inhibitor was found to suppress the transient activation of spinal ERK and reactivation of ERK in response to brushing post-incision, indicating that MEK may regulate, at least in part, incisional pain through MEK/ERK pathways.

\section{Acknowledgements}

The present study was supported by a grant from the National Natural Science Foundation of China (no. 81070897).

\section{References}

1. Dai RP, Li CQ, Zhang JW, Li F, Shi XD, Zhang JY, et al: Biphasic activation of extracellular signal-regulated kinase in anterior cingulate cortex distinctly regulates the development of pain-related anxiety and mechanical hypersensitivity in rats after incision. Anesthesiology 115: 604-613, 2011.

2. Cao H, Gao YJ, Ren WH, Li TT, Duan KZ, Cui YH, et al: Activation of extracellular signal-regulated kinase in the anterior cingulate cortex contributes to the induction and expression of affective pain. J Neurosci 29: 3307-3321, 2009.

3. Igaz LM, Winograd M, Cammarota M, Izquierdo LA, Alonso M, Izquierdo I, et al: Early activation of extracellular signal-regulated kinase signaling pathway in the hippocampus is required for short-term memory formation of a fear-motivated learning. Cell Mol Neurobiol 26: 989-1002, 2006.

4. Ji RR, Befort K, Brenner GJ and Woolf CJ: ERK MAP kinase activation in superficial spinal cord neurons induces prodynorphin and NK-1 upregulation and contributes to persistent inflammatory pain hypersensitivity. J Neurosci 22: 478-485, 2002.

5. Zhuang ZY, Gerner P, Woolf CJ and Ji RR: ERK is sequentially activated in neurons, microglia and astrocytes by spinal nerve ligation and contributes to mechanical allodynia in this neuropathic pain model. Pain 114: 149-159, 2005.

6. Costantini R, Affaitati G, Fabrizio A and Giamberardino MA: Controlling pain in the post-operative setting. Int J Clin Pharmacol Ther 49: 116-127, 2011.

7. Wu CL and Raja SN: Treatment of acute postoperative pain. Lancet 377: 2215-2225, 2011.

8. Zahn PK, Pogatzki-Zahn EM and Brennan TJ: Spinal administration of MK-801 and NBQX demonstrates NMDA-independent dorsal horn sensitization in incisional pain. Pain 114: 499-510, 2005.

9. Zahn PK, Subieta A, Park SS and Brennan TJ: Effect of blockade of nerve growth factor and tumor necrosis factor on pain behaviors after plantar incision. J Pain 5: 157-163, 2004.

10. Zhang L, Berta T, Xu ZZ, Liu T, Park JY and Ji RR: TNF-alpha contributes to spinal cord synaptic plasticity and inflammatory pain: distinct role of TNF receptor subtypes 1 and 2. Pain 152: 419-427, 2011.

11. Brennan TJ, Vandermeulen EP and Gebhart GF: Characterization of a rat model of incisional pain. Pain 64: 493-501, 1996. 
12. LoPachin RM, Rudy TA and Yaksh TL: An improved method for chronic catheterization of the rat spinal subarachnoid space. Physiol Behav 27: 559-561, 1981.

13. Chaplan SR, Bach FW, Pogrel JW, Chung JM and Yaksh TL: Quantitative assessment of tactile allodynia in the rat paw. $J$ Neurosci Methods 53: 55-63, 1994.

14. Ji RR, Gereau RW 4th, Malcangio M and Strichartz GR: MAP kinase and pain. Brain Res Rev 60: 135-148, 2009.

15. Ji RR, Baba H, Brenner GJ and Woolf CJ: Nociceptive-specific activation of ERK in spinal neurons contributes to pain hypersensitivity. Nat Neurosci 2: 1114-1119, 1999.

16. Fu D, Guo Q, Ai Y, Cai H, Yan J and Dai R: Glial activation and segmental upregulation of interleukin-1beta (IL-1beta) in the rat spinal cord after surgical incision. Neurochem Res 31: 333-340, 2006.

17. Samad TA, Moore KA, Sapirstein A, Billet S, Allchorne A Poole S, et al: Interleukin-lbeta-mediated induction of Cox-2 in the CNS contributes to inflammatory pain hypersensitivity. Nature 410: 471-475, 2001

18. Zheng W, Ouyang H, Zheng X, Liu S, Mata M, Fink DJ, et al: Glial TNFalpha in the spinal cord regulates neuropathic pain induced by HIV gp120 application in rats. Mol Pain 7: 40, 2011.
19. Andrade P, Visser-Vandewalle V, Hoffmann C, Steinbusch HW, Daemen MA and Hoogland G: Role of TNF-alpha during central sensitization in preclinical studies. Neurol Sci 32: 757-771, 2011.

20. Drdla-Schutting R, Benrath J, Wunderbaldinger $G$ and Sandkuhler J: Erasure of a spinal memory trace of pain by a brief, high-dose opioid administration. Science 335: 235-238, 2012.

21. Li CQ, Xu JM, Liu D, Zhang JY and Dai RP: Brain derived neurotrophic factor (BDNF) contributes to the pain hypersensitivity following surgical incision in the rats. Mol Pain 4: 27, 2008.

22. Cruz Duarte P, St-Jacques B and Ma W: Prostaglandin E2 contributes to the synthesis of brain-derived neurotrophic factor in primary sensory neuron in ganglion explant cultures and in a neuropathic pain model. Exp Neurol 234: 466-481, 2012.

23. Obata K, Yamanaka H, Dai Y, Mizushima T, Fukuoka T, Tokunaga A, et al: Activation of extracellular signal-regulated protein kinase in the dorsal root ganglion following inflammation near the nerve cell body. Neuroscience 126: 1011-1021, 2004. 